\title{
Evaluation of PDQ-8 and its relationship with PDQ-39 in China: a three-year longitudinal study
}

\author{
Kui Chen ${ }^{1 \dagger}$, Yu-Jie Yang ${ }^{1 \dagger}$, Feng-Tao Liu ${ }^{1,3}$, Da-Ke Li' ${ }^{1}$, Lu-Lu Bu${ }^{1}$, Ke Yang ${ }^{1}$, Ying Wang ${ }^{1}$, Bo Shen ${ }^{1}$, \\ Rong-Yuan Guan ${ }^{1}$, Jie Song ${ }^{1}$, Jian Wang ${ }^{1 *}$ and Jian-Jun $\mathrm{Wu}^{1,2^{*}}$
}

\begin{abstract}
Background: Parkinson's disease is characterized by motor and non-motor symptoms with wide ranging impacts on the health-related quality of life. The 39-item Parkinson's disease Questionnaire (PDQ-39) is the most widely used PD-specific health-related quality-of-life questionnaire. The short-form 8-item Parkinson's disease Questionnaire (PDQ-8) was found to produce results similar to that of the PDQ-39 cross-culturally. However, there is no evaluation of the PDQ-8 in the mainland of China.
\end{abstract}

Methods: In this longitudinal study, 283 patients with Parkinson's disease were recruited. The PDQ-39, the PDQ-8 and other scales were administered. Patients attended the clinic once annually for three years to complete the scales.

Results: The PDQ-8 was found to have good validity and reliability. There was a strong correlation between the summary indices of the PDQ-8 and the PDQ-39 $(r=0.93, P<0.001)$. Results suggested that the PDQ- 8 was also associated with other clinical scales of mobility, depression and cognition. The convergent validity and discriminant validity of the PDQ-8 were demonstrated by item-to-dimension correlations. There was acceptable internal consistency of the PDQ-8 (Cronbach's a: 0.80; Item-scale correlation efficient: 0.56-0.72). The PDQ-8 replicated the results of the PDQ-39 well at all follow-up time points (intraclass correlation coefficient: 0.96-0.98). In addition, there was good test-retest reliability of the PDQ-8.

Conclusion: The PDQ-8 is a valid and reliable instrument assessing health-related quality of life for PD patients in the mainland of China.

Keywords: Parkinson's disease, Health-related quality of life, PDQ-8 PDQ-39

\section{Background}

Patients with Parkinson's disease (PD) experience both motor disability and non-motor symptoms (NMS), which may affect their functioning and well-being $[1,2]$. During the past decade, increasing attention has been paid to health-related quality of life (HRQoL) of $\mathrm{PD}$ patients [3]. Thus, several scales have been developed to assess self-reported HRQoL in PD patients.

The 39-item Parkinson's disease Questionnaire (PDQ-39) is the most widely used PD-specific HRQoL questionnaire

\footnotetext{
* Correspondence: wangjian336@hotmail.com; wujianjun@fudan.edu.cn ${ }^{\dagger}$ Equal contributors

${ }^{1}$ Department of Neurology \& National Clinical Research Center for Aging and Medicine, Huashan Hospital, Fudan University, 12 Wulumuqi Zhong Road, Shanghai 200040, China

Full list of author information is available at the end of the article
}

and has been validated and translated cross-culturally $[4,5]$. It consists of 39 questions from 8 dimensions: mobility, activities of daily of living, emotional wellbeing, stigma, social support, cognition, communication, and bodily discomfort. To reduce the respondent burden, a brief version of PDQ-39, the 8-item Parkinson's disease Questionnaire (PDQ-8), was also developed [6]. The item with the strongest item-to-total correlation was selected from each dimension of the PDQ-39 to constitute the PDQ-8. The validity and reliability of the PDQ-8 have been demonstrated in several studies. In China, the PDQ39 has been translated and validated by Tsang KL et al. [7]. An evaluation of the Chinese version of PDQ-8 has been conducted in Taiwan with cross-sectional design and a relatively small sample size [8]. A different Chinese 
version of the PDQ-8 was also validated with promising results in Singapore [9]. Further analysis is needed for the generalized use of the PDQ-8 in China.

As a short form of the PDQ-39, the PDQ-8 is convenient for use in clinical settings. For example, the PDQ-8 was presented in the mPower study, an observational study that enrolled PD patients and that was conducted through an iPhone app interface [10]. Similarly, an online platform for clinical management of PD patients is being constructed for use in China at present. Comprehensive information will be collected in a selfreported way, which requires concise scales online to maintain the convenience and compliance of patients. Therefore, the PDQ- 8 is potentially more advantageous in this regard compared to the PDQ-39.

The present study assessed the validity and reliability of the Chinese version of PDQ-8, and compared it with the PDQ-39 in a longitudinal design in the mainland of China.

\section{Methods}

Subjects

Two hundred and eighty three PD patients were recruited from the Department of Neurology of Huashan hospital affiliated with Fudan University, Shanghai, China, between March 2012 and August 2012. Inclusion criteria were as follows: (1) a clinical diagnosis of PD according to the criteria of the United Kingdom PD Society Brain Bank [11]; (2) age 18 years or older; (3) consent to participate in the study. Subjects were excluded if they had cognitive impairment by the assessment of the mini-mental status examination (MMSE) (cutoff: < 24 for patients with education $>6$ years; <21 for patients with education 1-6 years; <17 for patients with no education) [12]. The patients with a history of neurological diseases other than PD, psychiatric disorders or surgical intervention, such as deep-brain stimulation of the subthalamic nucleus, were also excluded. The study was approved by the ethics committee of Huashan Hospital and all participants signed informed consent.

\section{Procedure and instruments}

Sociodemographic variables were collected including gender, age, age of onset, duration of disease, education and levodopa equivalent daily dose (LEDD). Disease severity and motor disability were evaluated by the Hoehn and Yahr stage (H\&Y) and the Unified Parkinson's Disease Rating Scale part III (UPDRS-III) [13] during the wearing off of levodopa effect (off phase: at least $12 \mathrm{~h}$ off-medications). Cognition was investigated with the MMSE [12]. The Beck Depression Inventory (BDI) [14] was also applied to participants to assess depression. During each follow-up, all of above scales were administered.
The Chinese version of PDQ-39 [7] measured the impact of PD on HRQoL of the patients in eight dimensions as mentioned previously. All of the items are scored on a 5-point ordinal scale ranging from 0 ("never") to 4 ("always"). Scores for each dimension range from 0 to 100, with higher scores indicating worse HRQoL. The PDQ-39 summary index (PDQ-39-SI) is summed over the eight dimensions and standardized from 0 to 100. Higher scores indicate worse HRQoL. The PDQ-8 summary index (PDQ-8-SI) is calculated from the following items of the PDQ-39: Q7, Q12, Q17, Q25, Q27, Q31, Q35 and Q37 with each item derived from one dimension. The PDQ-8-SI is standardized from 0 to 100, with higher scores indicating worse HRQoL as well.

In this study, we included the PDQ-8 items nested within the PDQ-39. Most of validation studies were performed in this way and have shown that the PDQ-8 can represent the original PDQ-39 well $[15,16]$. In addition, the PDQ-8 showed similar results when administered independently in PD samples compared with that when used nested in the PDQ-39 [17, 18].

\section{Statistical analysis}

Clinical data from all time points were subject to analysis. The median value and the inter-quartile range (IQR) were calculated to describe the distribution of continuous variables. Percentage was used to describe the distribution of category variables. Because data were non-normally distributed, the PDQ-39-SI and the PDQ-8-SI were compared by Wilcoxon test at baseline and follow-up years. Floor and ceiling effects of the two indices were assessed by means of frequencies.

The validity and reliability of the PDQ- 8 were assessed after calculating scores by the following methods:

Construct validity of the PDQ-8 was evaluated by correlating the PDQ-8-SI with disease specific scales including the PDQ-39-SI, the H\&Y staging and the UPDRS-III. The correlation between descriptive data such as age, duration of the disease, BDI, MMSE and PDQ-8-SI, was also examined. The principal component factor analysis (without rotation) was conducted for the PDQ-8. Since there was only one item in each dimension of the PDQ-8, the convergent and discriminant validity was examined according to the correlation coefficients among the items within the PDQ-8 and their parent subscales from the PDQ-39. If the item had a correlation coefficient with its own dimension above 0.40 and higher than with other dimensions, the convergent and discriminant validity of the PDQ-8 was considered acceptable.

Internal consistency was assessed by item-scale correlations and Cronbach's alpha. A correlation efficient above 
0.40 was considered acceptable [19], and Cronbach's alpha was considered satisfactory when it exceeded 0.70 [20].

At every time point, the concordance between the PDQ-8-SI and PDQ-39-SI was evaluated by the intraclass correlation coefficient (ICC; two-way mixed average, absolute agreement) in conjunction with the calculation of $95 \%$ confidence intervals (CIs). The correlation among the PDQ-8-SI, the PDQ-39-SI, H\&Y stage, UPDRS-III and BDI was also evaluated. Test-retest reliability was evaluated by Wilcoxon tests between PDQ-8-SI of different time points.

All correlations between variables in this study were calculated by Spearman's Rho test, since data were nonnormally distributed. The strength of correlations was determined by Cohen's criteria [21], where strong and moderate correlations were described as $\geq 0.50$ and $0.30-0.49$, respectively. The significance level was set at $P<0.05$. Data were analyzed using SPSS 22.0 for Windows.

\section{Results}

\section{Patients' demographic data}

Two hundred and eighty three subjects were recruited at baseline (Table 1). The majority of patients were male (58.7\%) and had a median age of 57 years (IQR: 45-64). The median of age at onset and disease duration were 51 years (IQR: $41-60)$ and 36 months (IQR: 18-67) respectively. $51.1 \%$ patients completed 7-12 years of education. The patients' median LEDD was $300 \mathrm{mg}$ (IQR: 75-451). Disease severity in participants was measured by the H\&Y stage, with $44.0 \%$ of patients at stage 2 . Mobility disability was evaluated by UPDRS-III and showed a median score of 30 (IQR: 20-38). BDI and MMSE were also assessed as described in Table 1 . The median values of the PDQ-39-SI and the PDQ-8-SI were 17.3 (IQR: 9.0-27.6) and 18.8 (IQR: 9.4-31.3) without significant difference $(P=0.851)$. There was no evidence of floor or ceiling effects on the PDQ-8-SI, with only 17 (6.0\%) participants scoring zero and no participants scoring the maximum of 100 on the instrument. However, floor effects could be found on the dimensions of social support and communication on the PDQ-39.

In the first and second follow-up year, 101 (35.7\%) and $81(28.6 \%)$ patients remained in the study respectively. In the first year, there were no significant differences between age, duration of disease, gender, MMSE, BDI, PDQ-8-SI and PDQ-39-SI in the follow-up patients and dropouts. However, the dropouts had earlier age at onset $(P=0.045)$, lower level of education $(P=0.046)$, higher H\&Y stage $(P=0.040)$, higher UPDRS-III scores $(P=0.001)$ and a higher levodopa dose $(P=0.030)$. In the second year, there were no significant differences between gender, MMSE, BDI PDQ-8-SI and PDQ-39-SI in the follow-up patients and dropouts. Age, duration of
Table 1 Demographic information of participants at baseline $(N=283)$

\begin{tabular}{|c|c|c|c|}
\hline & Median & $\begin{array}{l}\text { Quartile/ } \\
\text { Percentage }\end{array}$ & $\begin{array}{l}\text { Floor/ } \\
\text { Ceiling \% }\end{array}$ \\
\hline Gender (male) & 166 & $58.7 \%$ & \\
\hline Age (year) & 57 & $45-64$ & \\
\hline Age of onset (year) & 51 & $41-60$ & \\
\hline Duration of disease (month) & 36 & $18-67$ & \\
\hline \multicolumn{4}{|l|}{ Education (year) } \\
\hline$<7$ & 29 & $10.2 \%$ & \\
\hline $7-12$ & 145 & $51.1 \%$ & \\
\hline$>12$ & 109 & $38.5 \%$ & \\
\hline \multicolumn{4}{|l|}{ H\&Y stage } \\
\hline 1 & 71 & $25.1 \%$ & \\
\hline 2 & 130 & $45.9 \%$ & \\
\hline 3 & 73 & $25.7 \%$ & \\
\hline 4 & 7 & $2.5 \%$ & \\
\hline 5 & 2 & $0.7 \%$ & \\
\hline UPDRS-III & 30 & $20-38$ & \\
\hline LEDD (mg) & 300 & $75-451$ & \\
\hline $\mathrm{BDI}$ & 11 & $6.0-18.0$ & \\
\hline MMSE & 28 & $27-29$ & \\
\hline PDQ-8-SI & 18.8 & $9.4-31.3$ & $6.0 \% / 0$ \\
\hline PDQ-39-SI & 17.3 & $9.0-27.6$ & $0.4 \% / 0$ \\
\hline Mobility & 15 & $5.0-30.0$ & $14.5 \% / 0$ \\
\hline Activity of daily living & 12.5 & $0.0-28.2$ & $25.8 \% / 0$ \\
\hline Emotional well-being & 16.7 & $8.3-33.3$ & $15.5 \% / 0.4 \%$ \\
\hline Stigma & 18.8 & $0.0-37.5$ & $25.1 \% / 2.5 \%$ \\
\hline Social support & 0 & $0.0-8.3$ & $63.6 \% / 0.4 \%$ \\
\hline Cognitions & 18.8 & $12.5-36.0$ & $11.7 \% / 0.4 \%$ \\
\hline Communication & 8.3 & $0.0-25.0$ & $45.9 \% / 0.4 \%$ \\
\hline Bodily discomfort & 25 & $8.3-41.7$ & $18.7 \% / 0.7 \%$ \\
\hline
\end{tabular}

Abbreviations: BDI Beck Depression Inventory, H\&Y stage Hoehn and Yahr stage, LEDD Levodopa equivalent daily dose, MMSE Mini-Mental Status Examination, $P D Q-8$ 8-item Parkinson's disease Questionnaire, PDQ-8-SI PDQ-8 summary index, $P D Q-39$ 39-item Parkinson's disease Questionnaire, $P D Q-39-S I$ PDQ-39 summary index, UPDRS-III Unified Parkinson's Disease Rating Scale part III

disease, age at onset,education,LEDD, H\&Y stage and UPDRS-III scores were different between groups $(P<0.05)$.

\section{Validity of the PDQ-8}

Construct validity was explored by correlating the PDQ-8-SI with disease specific scales including the PDQ-39-SI, H\&Y stage and UPDRS-III (Table 2). Results showed that the PDQ-8-SI and the PDQ-39-SI were highly correlated $(r=0.93, P<0.001)$. There were moderate correlations among the $H \& Y$ stage, the UPDRS-III and the PDQ-8-SI with coefficient of 0.48 and 0.47 respectively. In addition, the PDQ-8-SI 
Table 2 Correlation matrix for criterion validity

\begin{tabular}{ll}
\hline & Correlation with PDQ-8-SI \\
\hline PDQ-39-SI & $0.93^{* *}$ \\
Age of onset & $-0.19^{* *}$ \\
Duration of disease & $0.31^{* *}$ \\
Education & $-0.19^{*}$ \\
H\&Y stage & $0.48^{* *}$ \\
UPDRS-III & $0.47^{* *}$ \\
LEDD & $0.32^{* *}$ \\
BDI & $0.67^{* *}$ \\
MMSE & $-0.20^{* *}$ \\
\hline
\end{tabular}

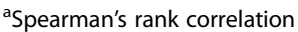

** $P<0.001$; $P<0.01$

Abbreviations: BDI Beck Depression Inventory, H\&Y stage Hoehn and Yahr stage, LEDD Levodopa equivalent daily dose, MMSE Mini-Mental Status Examination, $P D Q-8$ 8-item Parkinson's disease Questionnaire, $P D Q-8-S /$ PDQ-8 summary index, $P D Q-39$ 39-item Parkinson's disease Questionnaire, $P D Q-39-S I$ PDQ-39 summary index, UPDRS-III Unified Parkinson's Disease Rating Scale part III

was strongly correlated with the BDI $(r=0.67$, $P<0.001)$ and weakly correlated with the MMSE $(r=-0.20, P<0.001)$. Additionally, there were disease associated variables that correlated with the PDQ-8-SI, weekly or moderately, such as age at onset, disease duration, education and LEDD.

For the PDQ-8, a one-factor solution was derived from principal component factor analysis, suggesting the 8 items of the instrument can be summed to generate a single index score (Table 3 ). There was only one factor with an eigenvalue greater than 1.0 (3.4) accounting for $42.86 \%$ of the total variance in the PDQ- 8 scores. The loadings of the 8 items on the single factor ranged from 0.57 to 0.79 .

In tests of item convergent validity, the PDQ-8 items were strongly correlated with their own dimensions in the PDQ-39 ( $r=0.71-0.87)$ (Table 4$)$. In tests of item discriminant validity, all of the PDQ-8 items were

Table 3 Principal component analysis of the 8 items of PDQ-8

\begin{tabular}{lll}
\hline Dimension & PDQ-8 item & Factor loadings \\
\hline Mobility & Q7 & 0.59 \\
Activity of daily living & Q12 & 0.62 \\
Emotional well-being & Q17 & 0.79 \\
Stigma & Q25 & 0.68 \\
Social support & Q27 & 0.61 \\
Cognitions & Q31 & 0.57 \\
Communication & Q35 & 0.77 \\
Bodily discomfort & Q37 & 0.58 \\
\% Variance explained & & $42.86 \%$ \\
Component eigenvalue & & 3.4
\end{tabular}

Principal component factor analysis (without rotation) Abbreviations: PDQ-8 8-item Parkinson's disease Questionnaire
Table 4 Item-to-dimension correlations

\begin{tabular}{llll}
\hline $\begin{array}{l}\text { PDQ-39 } \\
\text { dimension }\end{array}$ & $\begin{array}{l}\text { PDQ-8 } \\
\text { item }\end{array}$ & $\begin{array}{l}\text { Item to own- } \\
\text { dimension } \\
\text { correlation }\end{array}$ & $\begin{array}{l}\text { Item to other } \\
\text { dimensions } \\
\text { correlation }^{\text {a }}\end{array}$ \\
\hline Mobility & Q7 & 0.80 & $0.30-0.43$ \\
Activity of daily living & Q12 & 0.71 & $0.23-0.50$ \\
Emotional well-being & Q17 & 0.87 & $0.33-0.53$ \\
Stigma & Q25 & 0.85 & $0.24-0.49$ \\
Social support & Q27 & 0.83 & $0.26-0.46$ \\
Cognitions & Q31 & 0.72 & $0.21-0.44$ \\
Communication & Q35 & 0.85 & $0.40-0.60$ \\
Bodily discomfort & Q37 & 0.84 & $0.24-0.39$ \\
\hline
\end{tabular}

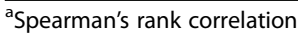

$P<0.001$ in each case

Abbreviations: $P D Q-8$ 8-item Parkinson's disease Questionnaire, $P D Q-39$

39-item Parkinson's disease Questionnaire

correlated higher with their own dimensions than with any other item.

\section{Reliability of the PDQ-8}

In the PDQ-8, the item-total correlation coefficients ranged from 0.56 to 0.72 (Table 5 ). The item of activity of daily of living showed the lowest item-total correlation, whereas the item of emotional well-being presented the highest. The internal reliability of the eight items of the PDQ-8 was calculated using the Cronbach's alpha statistic. Estimates were generally regarded as acceptable in excess of 0.70 .

At all follow up time points, the PDQ-39, the PDQ-8, H\&Y stage, UPDRS-III and BDI were calculated, and the correlations among these scores were assessed (Table 6). There were no significant differences between the PDQ39-SI and the PDQ-8-SI at any of the time points. The mean differences between the two scores were small, ranging from 0 to -1.1 , with ICCs ranging from 0.96 to 0.98 at three time points. The PDQ-8-SI and the PDQ39-SI correlated with the H\&Y and the UPDRS-III

Table 5 Internal consistency

\begin{tabular}{lllc}
\hline Scales/subscales & Items & Item-scale correlation $^{a}$ & Cronbach's a $^{\text {PDQ-8 }}$ \\
Mobility & Q7 & $0.56-0.72$ & 0.80 \\
Activity of daily living & Q12 & 0.56 & \\
Emotional well-being & Q17 & 0.72 \\
Stigma & Q25 & 0.66 \\
Social support & Q27 & 0.57 \\
Cognitions & Q31 & 0.57 \\
Communication & Q35 & 0.70 \\
Bodily discomfort & Q37 & 0.61 \\
\hline
\end{tabular}

${ }^{\mathrm{a}}$ Spearman's rank correlation

$P<0.001$ in each case

Abbreviations: PDQ-8 8-item Parkinson's disease Questionnaire 
Table 6 Descriptive statistics for PDQ-39-SI and PDQ-8-SI at baseline and follow up

\begin{tabular}{|c|c|c|c|c|c|c|c|c|c|c|c|}
\hline & $\mathrm{N}$ & Mean & SD & Median & 25th percentile & 75th percentile & $\begin{array}{l}\text { Correlation } \\
\text { with } H \& Y^{\mathrm{a}}\end{array}$ & $\begin{array}{l}\text { Correlation } \\
\text { with UPDRS-III }\end{array}$ & $\begin{array}{l}\text { Correlation } \\
\text { with } \mathrm{BDI}^{\mathrm{a}}\end{array}$ & Average ICC & $95 \% \mathrm{Cl}$ \\
\hline Baseline & 283 & & & & & & & & & & \\
\hline PDQ-39-SI & & 20.2 & 14.6 & 17.3 & 9.0 & 27.6 & $0.53^{* *}$ & $0.50^{* *}$ & $0.67^{* *}$ & & \\
\hline PDQ-8-SI & & 21.3 & 16.8 & 18.8 & 9.4 & 31.3 & $0.48^{* *}$ & $0.47^{* *}$ & $0.64^{* *}$ & $0.96^{* *}$ & $0.95-0.97$ \\
\hline Difference & & -1.1 & -2.2 & -1.5 & -0.4 & -3.7 & & & & & \\
\hline Follow-up year 1 & 101 & & & & & & & & & & \\
\hline PDQ-39-SI & & 17.5 & 13.3 & 14.1 & 8.3 & 24 & $0.35^{* *}$ & $0.40^{* *}$ & $0.68^{* *}$ & & \\
\hline PDQ-8-SI & & 17.5 & 14.9 & 15.6 & 6.3 & 21.9 & $0.29^{* *}$ & $0.34^{* *}$ & $0.69^{* *}$ & $0.96^{* *}$ & $0.95-0.98$ \\
\hline Difference & & 0 & -1.6 & -1.5 & 2 & 2.1 & & & & & \\
\hline Follow-up year 2 & 81 & & & & & & & & & & \\
\hline PDQ-39-SI & & 19.7 & 16 & 14.7 & 9.0 & 26.3 & $0.38^{* *}$ & $0.43^{* *}$ & $0.66^{* *}$ & & \\
\hline PDQ-8-SI & & 19.9 & 17.5 & 15.6 & 6.3 & 28.1 & $0.32^{* *}$ & $0.43^{* *}$ & $0.69^{* *}$ & $0.98^{* *}$ & $0.97-0.99$ \\
\hline Difference & & -0.2 & -1.5 & -0.9 & 2.7 & -1.8 & & & & & \\
\hline
\end{tabular}

${ }^{\mathrm{a}}$ Spearman's rank correlation

** $P<0.001 ; * P<0.01$

Abbreviations: BDI Beck Depression Inventory, $H \& Y$ stage Hoehn and Yahr stage, ICC intraclass correlation coefficient, $P D Q-8$ 8-item Parkinson's disease Questionnaire, $P D Q-8-S I$ PDQ-8 summary index, PDQ-39 39-item Parkinson's disease Questionnaire, PDQ-39-SI PDQ-39 summary index, SD standard deviation, UPDRS-III Unified Parkinson's Disease Rating Scale part III

moderately and correlated with the BDI strongly in all follow-up years.

For test-retest reliability, we chose the participants who completed the questionnaires in two time points to compose three groups (Additional file 1: Table S1). There was no significant difference between the PDQ-39-SI of any two time points in the follow up, which stood for the stable quality of life of three groups. We then compared the results of the PDQ-8-SI assessments across the different years and found no significant difference.

\section{Discussion}

To our knowledge, this was the first study to evaluate the PDQ-8 in mainland China longitudinally with a large sample. Our results showed good validity and reliability of the Chinese version PDQ-8, in accordance with other validation studies $[8,22]$. There was no floor or ceiling effect of the PDQ-8-SI and the PDQ-39-SI. However, significant floor effects could be observed on dimensions of social support and communication, with similar results in studies of other countries [22, 23]. This may result from the small number of items in these two dimensions and the higher social participation of subjects consenting to this study. In addition, the median age at onset was 51 years in this study, which was younger than 60 , the typical reported age at onset [24]. This finding could be a result from the high level of attention given to the early onset PD patients in our clinical practice, especially in patients considered to have genetic causes.

In this study, the validity of the PDQ-8 was confirmed. The comparison was made between the PDQ-8-SI and the PDQ-39-SI, and the results suggest that the PDQ8-SI closely replicates the PDQ-39-SI. Moreover, the PDQ-8-SI correlated moderately to the standardized and specific measures of motor disability (H\&Y stage and UPDRS-III), indicating that worse mobility was associated with poorer HRQoL [25]. In the analysis of the correlation between the PDQ-8-SI and some other clinical parameters, we found that the BDI has a strong correlation with the PDQ-8-SI, a result that is consistent in other studies correlating depression and the PDQ-39-SI [26-28]. This finding is in agreement with the conclusions of other studies reporting that depression negatively contributes to the daily life of PD patients supported by it being consistently linked to poor HRQoL [29, 30]. Via factor analysis of the PDQ-8, the summary index was reasonable and useful in assessing the HRQoL. These results support the construct validity of using the PDQ-8 in China. In addition, convergent validity and discriminant validity were present with higher item to own-dimension correlations and lower Item to other-dimension correlations. Furthermore, we found that disease duration, education, mobility disability, LEDD, depression and cognition were correlated with the PDQ-8-SI, which indicates that these items play a role in the HRQoL of PD patients. This reminds us to pay more attention to patients with longer disease duration, less education, higher daily levodopa dosage, lower mood and poorer cognition and mobility, and try to optimize their care individually in addition to medical treatment alone. 
Our findings support the reliability of the Chinese version of PDQ-8. The results produced Cronbach's alpha of 0.80 and item-total correlations ranging from 0.56 to $0.72(P<0.001)$, approximating the results reported in the studies of Taiwan and Singapore [8, 31]. The PDQ-8-SI had ICCs ranging between 0.96 and 0.98 and agreed with the PDQ-39 in each follow-up year, suggesting that the PDQ- 8 is sufficiently reliable for its use in the longitudinal evaluation of PD patients. In the follow up, the correlations between the BDI and the PDQ-8-SI were consistently stronger than that between scales of mobility and the PDQ- 8 , which indicates that the depression did contribute more to the HRQoL than the motor symptoms. Hence, this points to the importance of including methods to manage the psychological needs of patients as part of their overall care strategy.

Despite our positive report, there are some limitations to this study that warrant discussion. Firstly, the number of patients decreased by $64.3 \%$ and $71.4 \%$ respectively between enrollment and each follow-up. Documented reasons for patients' refusal to attend follow-up visits included, busy with work and travel. Clearly, if we had less patients dropped out, a more reliable results would have been produced. Secondly, the test-retest interval could have been too long in this study, allowing for the effect of disease progression and environmental changes to impact on quality of life over the one-year period. In hindsight, we believe that a one-month interval would be better. While we have shown that usage of the PDQ8 is sufficient, the level of resolution captured using the PDQ-39 is far greater.

\section{Conclusion}

In conclusion, the PDQ-8 is an instrument to more quickly assess HRQoL based on a patients' own report. It can be used in the busy clinical setting with less time and acceptable accuracy helping to ease administration, reduce respondent burden and improve medical decision making, in particular when conducting a longitudinal study.

\section{Additional file}

Additional file 1: Table S1. Comparison of PDQ-39-SI and PDQ-8-SI at different time points. (DOCX $23 \mathrm{~kb}$ )

\footnotetext{
Abbreviations

BDI: Beck Depression Inventory; Cl: Confidence intervals; H \& Y: Hoehn and Yahr stage; HRQoL: Health-related quality of life; ICC: Intraclass correlation coefficient; IQR: Inter-quartile range; LEDD: Levodopa equivalent daily dose; MMSE: Mini-Mental Status Examination; NMS: Non-motor symptoms; PD: Parkinson's disease; PDQ-39: 39-item Parkinson's disease Questionnaire; PDQ-39-SI: PDQ-39 summary index; PDQ-8: 8-item Parkinson's disease Questionnaire; PDQ-8-SI: PDQ-8 summary index; SD: Standard deviation; UPDRS-III: Unified Parkinson's Disease Rating Scale part III
}

\section{Acknowledgements}

We would like to thank all those who contributed to our research particularly the patients who participated in the study, and Dr. James B. Koprich for editing the manuscript.

\section{Funding}

This work was supported by grants to Jian Wang, Jian-Jun Wu and Feng-Tao Liu from the National Natural Science Foundation of China (81,571,232 and 81,371,413), the National Key R\&D Program of China (2016YFC1306500) from Ministry of Science and Technology of China, project from the Science and Technology Commission of Shanghai Municipality (15ZR1435800, JA2015-Z002), and Scientific Research Project (2016QD01) from Huashan Hospital affiliated to Fudan University.

\section{Availability of data and materials}

The datasets used and analysed during the current study are available from the corresponding author on reasonable request.

\section{Authors' contributions}

(1) CK, YYJ, WJJ and WJ were responsible for the conception and design of the study. LFT, LDK, BLL, YK, WY and SJ contributed to acquisition of data. CK, YYJ, SB and GRY were involved in analysis and interpretation of data. (2) CK drafted the article. CK, YYJ and WJJ revised it critically for important intellectual content. (3) All authors gave final approval of the version to be submitted.

\section{Author's information}

Not applicable.

\section{Ethics approval and consent to participate}

The study was approved by the ethics committee of Huashan Hospital and all participants signed informed consent.

\section{Consent for publication}

Not applicable.

\section{Competing interests}

The authors declare that they have no competing interests.

\section{Publisher's Note}

Springer Nature remains neutral with regard to jurisdictional claims in published maps and institutional affiliations.

\section{Author details \\ ${ }^{1}$ Department of Neurology \& National Clinical Research Center for Aging and Medicine, Huashan Hospital, Fudan University, 12 Wulumuqi Zhong Road, Shanghai 200040, China. 'Department of Neurology, Jing'an District Centre Hospital of Shanghai, Shanghai, China. ${ }^{3}$ Department of Neurology, Huashan Hospital North, Fudan University, Shanghai, China.}

Received: 27 March 2017 Accepted: 16 August 2017

Published online: 24 August 2017

\section{References}

1. Chaudhuri KR, Odin P. The challenge of non-motor symptoms in Parkinson's disease. Prog Brain Res. 2010;184:325-41.

2. Martinez-Martin P, Schapira AH, Stocchi F, Sethi K, Odin P, MacPhee G, et al. Prevalence of nonmotor symptoms in Parkinson's disease in an international setting; study using nonmotor symptoms questionnaire in 545 patients. Mov Disord. 2007;22:1623-9.

3. van Uem JM, Marinus J, Canning C, van Lummel R, Dodel R, LiepeltScarfone I, et al. Health-related quality of life in patients with Parkinson's disease-a systematic review based on the ICF model. Neurosci Biobehav Rev. 2016;61:26-34.

4. Bushnell DM, Martin ML. Quality of life and Parkinson's disease: translation and validation of the US Parkinson's disease questionnaire (PDQ-39). Qual Life Res. 1999;8:345-50.

5. Hagell P, Whalley D, McKenna SP, Lindvall O. Health status measurement in Parkinson's disease: validity of the PDQ-39 and Nottingham health profile. Mov Disord. 2003;18:773-83. 
6. Peto V, Jenkinson C, Fitzpatrick R. PDQ-39: a review of the development, validation and application of a Parkinson's disease quality of life questionnaire and its associated measures. J Neurol. 1998;245(Suppl 1):S10-4.

7. Tsang KL, Chi I, Ho SL, Lou WW, Lee TM, Chu LW. Translation and validation of the standard Chinese version of PDQ-39: a quality-of-life measure for patients with Parkinson's disease. Mov Disord. 2002;17:1036-40.

8. Huang TT, Hsu HY, Wang BH, Chen KH. Quality of life in Parkinson's disease patients: validation of the short-form eight-item Parkinson's disease questionnaire (PDQ-8) in Taiwan. Qual Life Res. 2011;20:499-505.

9. Luo N, Tan LC, Zhao Y, Lau PN, Au WL, Li SC. Determination of the longitudinal validity and minimally important difference of the 8-item Parkinson's disease questionnaire (PDQ-8). Mov Disord. 2009;24:183-7.

10. Bot BM, Suver C, Neto EC, Kellen M, Klein A, Bare C, et al. The mPower study. Parkinson disease mobile data collected using ResearchKit Scientific data. 2016;3:160011.

11. Hughes AJ, Daniel SE, Kilford L, Lees AJ. Accuracy of clinical diagnosis of idiopathic Parkinson's disease: a clinico-pathological study of 100 cases. J Neurol Neurosurg Psychiatry. 1992;55:181-4.

12. Li H, Jia J, Yang Z. Mini-mental state examination in elderly Chinese: a population-based normative study. Journal of Alzheimer's disease : JAD. 2016;53:487-96.

13. Goetz CG, Poewe W, Rascol O, Sampaio C, Stebbins GT, Counsell C, et al. Movement Disorder Society task force report on the Hoehn and Yahr staging scale: status and recommendations. Mov Disord. 2004;19:1020-8.

14. Shek DT. Reliability and factorial structure of the Chinese version of the Beck depression inventory. J Clin Psychol. 1990;46:35-43.

15. Jenkinson C, Fitzpatrick R. Cross-cultural evaluation of the short form 8-item Parkinson's disease questionnaire (PDQ-8): results from America, Canada, Japan. Italy and Spain Parkinsonism Relat Disord. 2007:13:22-8.

16. Katsarou Z, Bostantjopoulou S, Peto V, Kafantari A, Apostolidou E, Peitsidou E. Assessing quality of life in Parkinson's disease: can a short-form questionnaire be useful? Mov Disord. 2004;19:308-12.

17. Fereshtehnejad SM, Naderi N, Rahmani A, Shahidi GA, Delbari A, Lokk J. Psychometric study of the Persian short-form eight-item Parkinson's disease questionnaire (PDQ-8) to evaluate health related quality of life (HRQoL). Health Qual Life Outcomes. 2014;12:78.

18. Franchignoni F, Giordano A, Ferriero G. Rasch analysis of the short form 8-item Parkinson's disease questionnaire (PDQ-8). Qual Life Res. 2008; 17:541-8.

19. Fayers PM, Machin D. Quality of life: assessment, analysis and interpretation. Chichester: John Wiley \& Sons; 2000.

20. Nunnally JC, Bernstein IH. Psychometric theory (3rd edn). New York: McGraw-Hill; 1994.

21. Cohen J. A power primer. Psychol Bull. 1992;112:155-9.

22. Luo N, Tan LC, Li SC, Soh LK, Thumboo J. Validity and reliability of the Chinese (Singapore) version of the Parkinson's disease questionnaire (PDQ-39). Qual Life Res. 2005;14:273-9.

23. Peto $V$, Jenkinson $C$, Fitzpatrick $R$, Greenhall $R$. The development and validation of a short measure of functioning and well being for individuals with Parkinson's disease. Qual Life Res. 1995:4:241-8.

24. Katzenschlager R, Head J, Schrag A, Ben-Shlomo Y, Evans A, Lees AJ. Fourteen-year final report of the randomized PDRG-UK trial comparing three initial treatments in PD. Neurology. 2008;71:474-80.

25. Gomez-Esteban JC, Zarranz JJ, Lezcano E, Tijero B, Luna A, Velasco F, et al. Influence of motor symptoms upon the quality of life of patients with Parkinson's disease. Eur Neurol. 2007:57:161-5.

26. Slawek J, Derejko M, Lass P. Factors affecting the quality of life of patients with idiopathic Parkinson's disease-a cross-sectional study in an outpatient clinic attendees. Parkinsonism Relat Disord. 2005:11:465-8.

27. Jones JD, Butterfield LC, Song W, Lafo J, Mangal P, Okun MS, et al. Anxiety and depression are better correlates of Parkinson's disease quality of life than apathy. J Neuropsychiatry Clin Neurosci. 2015;27:213-8.

28. Muller B, Assmus J, Herlofson K, Larsen JP, Tysnes OB. Importance of motor vs. non-motor symptoms for health-related quality of life in early Parkinson's disease. Parkinsonism Relat Disord. 2013;19:1027-32.
29. Pontone GM, Bakker CC, Chen S, Mari Z, Marsh L, Rabins PV, et al. The longitudinal impact of depression on disability in Parkinson disease. Int J Geriatr Psychiatr. 2016;31:458-65.

30. Carod-Artal FJ, Ziomkowski S, Mourao Mesquita H, Martinez-Martin P. Anxiety and depression: main determinants of health-related quality of life in Brazilian patients with Parkinson's disease. Parkinsonism Relat Disord. 2008;14:102-8.

31. Tan LC, Lau PN, Au WL, Luo N. Validation of PDQ-8 as an independent instrument in English and Chinese. J Neurol Sci. 2007;255:77-80.

\section{Submit your next manuscript to BioMed Central and we will help you at every step:}

- We accept pre-submission inquiries

- Our selector tool helps you to find the most relevant journal

- We provide round the clock customer support

- Convenient online submission

- Thorough peer review

- Inclusion in PubMed and all major indexing services

- Maximum visibility for your research

Submit your manuscript at www.biomedcentral.com/submit
) Biomed Central 